\title{
Abkürzungen und allgemeine Referenzwerke
}

BNJ (ab 2007):

DKP (1979):

DNP (1996-2003):

Fajen u. Wacht (2008):

FGrH (1923-1958/

1954-1969):

Lampe (1961):

LfgrE (1979-2010):

LIMC (1981-1999):

LSJ ( $\left({ }^{10} 1994\right):$

LThK (2009):

OCD ( $\left.{ }^{4} 2012\right):$

ODB (1991):

Peek (1968-1975):

$R E(1893-1980):$

Roscher (1886-

1937/31992-1993):
Brill's New Jacoby, Edited by lan Worthington, http://referenceworks.brillonline.com/browse/brill-s-new-jacoby (Stand: 27.7.2015).

Der Kleine Pauly. Lexikon der Antike, auf der Grundlage von Paulys Realencyclopädie der classischen Altertumswissenschaft, unter Mitwirkung zahlreicher Fachgelehrter bearbeitet und herausgegeben von Konrat Ziegler u. Walther Sontheimer, 5 Bde., München (Nachdruck der Ausgabe von 1964-1975; dtv 5963).

Der Neue Pauly. Enzyklopädie der Antike, herausgegeben von Hubert Cancik u. Helmut Schneider, 16 Bde., Stuttgart u. Weimar.

Fritz Fajen u. Manfred Wacht, Concordantia Nonni Dionysiacorum. Konkordanz zu den Dionysiaka des Nonnos, 5 Bde., Hildesheim, Zürich u. New York (Alpha-Omega. Reihe A, Lexika, Indizes, Konkordanzen zur klassischen Philologie 251).

Die Fragmente der griechischen Historiker, begründet von Felix Jacoby, Leiden u. a.

A Patristic Greek Lexicon, Edited by Geoffrey W. H. Lampe, Oxford u. a. Lexikon des frühgriechischen Epos, begründet von Bruno Snell, im Auftrag der Akademie der Wissenschaften in Göttingen vorbereitet und herausgegeben vom Thesaurus Linguae Graecae, Göttingen, Bd. 1: A (1979), Bd. 2: $B-\Lambda$ (1991), Bd. 3: $M-\Pi$ (2004), Bd. 4: $P-\Omega$ (2010).

Lexicon Iconographicum Mythologiae Classicae, publié par la Fondation pour le Lexicon Iconographicum Mythologiae Classicae, rédaction Hans Christoph Ackermann et Jean-Robert Gisler, Bd. I, 1-VIII, $2+2$ Bde. Indices, Zürich u. München.

A Greek-English Lexicon, with a Supplement 1968, Compiled by Henry George Liddell and Robert Scott, Revised and Augmented by Henry Stuart Jones with the Assistance of Roderick McKenzie, Oxford u. a.

Lexikon für Theologie und Kirche, begründet von Michael Buchberger, herausgegeben von Walter Kasper mit Konrad Baumgartner, Horst Bürkle, Klaus Ganzer, Karl Kertelge, Wilhelm Korff u. Peter Walter, Freiburg i. Br. u. a. (durchgesehene Ausgabe der 3. Auflage 1993-2001).

The Oxford Classical Dictionary, Edited by Simon Hornblower, Anthony Spawforth u. Esther Eidinow, Oxford u. a.

The Oxford Dictionary of Byzantium, Prepared at Dumbarton Oaks by Alexander P. Každan, 3 Bde., New York u. a.

Lexikon zu den Dionysiaka, herausgegeben von einer Arbeitsgruppe des Instituts für Klassische Philologie an der Martin-Luther-Universität HalleWittenberg unter der Leitung von Werner Peek, Bd. 1 (1968), Bd. 2 (1973), Bd. 3 (1974), Bd. 4 (1975), Hildesheim (Alpha-Omega. Lexika, Indizes, Konkordanzen zur Klassischen Philologie 3).

Paulys Real-Encyclopädie der classischen Altertumswissenschaft, neue Bearbeitung, unter Mitwirkung zahlreicher Fachgenossen herausgegeben von Georg Wissowa u. Wilhem Kroll, erste Reihe: Bd. I,1-XXIV: A-Q; zweite Reihe: Bd. I,A,1: $R-Z$; Suppl. I-XVI, Register, Stuttgart u. a.

Ausführliches Lexikon der griechischen und römischen Mythologie, herausgegeben von Wilhelm Heinrich Roscher, 6 Bde. + 4 Suppl., Leipzig. 
SSH (2005):

Ueding (1992-2012):
Supplementum Supplementi Hellenistici, herausgegeben von Hugh LloydJones, Berlin u. New York (Texte und Kommentare 26).

Historisches Wörterbuch der Rhetorik, herausgegeben von Gert Ueding, mitbegründet von Walter Jens, 10 Bde., Darmstadt. 